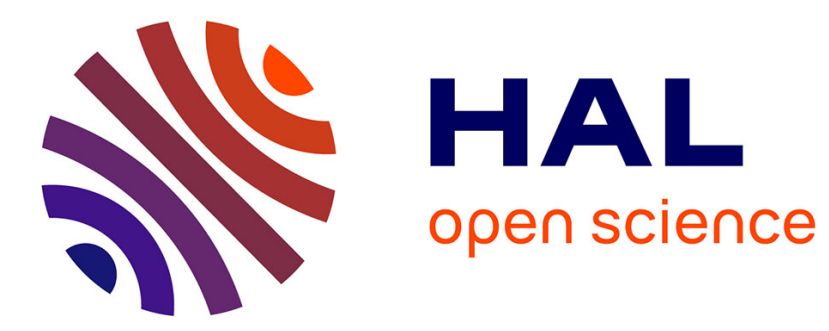

\title{
Strange wave formation and detonation onset in narrow channels
}

\author{
Yves Ballossier, Florent Virot, J. Melguizo-Gavilanes
}

\section{To cite this version:}

Yves Ballossier, Florent Virot, J. Melguizo-Gavilanes. Strange wave formation and detonation onset in narrow channels. Journal of Loss Prevention in the Process Industries, 2021, 72, pp.104535. 10.1016/j.jlp.2021.104535 . hal-03412012

\section{HAL Id: hal-03412012 \\ https://hal.science/hal-03412012}

Submitted on 16 Nov 2021

HAL is a multi-disciplinary open access archive for the deposit and dissemination of scientific research documents, whether they are published or not. The documents may come from teaching and research institutions in France or abroad, or from public or private research centers.
L'archive ouverte pluridisciplinaire HAL, est destinée au dépôt et à la diffusion de documents scientifiques de niveau recherche, publiés ou non, émanant des établissements d'enseignement et de recherche français ou étrangers, des laboratoires publics ou privés. 


\title{
Strange wave formation and detonation onset in narrow channels
}

\author{
Yves Ballossier*, Florent Virot** and Josué Melguizo-Gavilanes** \\ Institut Pprime, UPR 3346 CNRS, ISAE-ENSMA, Futuroscope Chasseneuil, France
}

\section{ARTICLE INFO}

\section{Keywords:}

DDT

narrow smooth channels

hydrogen safety

flow visualization

strange wave

\begin{abstract}
A B S T R ACT
Experiments are conducted in a smooth $10 \times 10 \mathrm{~mm}$ square cross-section, 1-m long channel, closed at the ignition end and open at the other end. Simultaneous two-direction schlieren visualization is used to investigate the three-dimensional dynamics of transition to detonation for a stoichiometric $\mathrm{H}_{2}-\mathrm{O}_{2}$ mixture. Results show the existence of two distinct structures before detonation onset: (i) asymmetric, composed of an oblique shock trailed by a flame, that runs preferentially along the wall, and seems to get ignited inside the boundary layer developed by the precursor shock; (ii) symmetric, referred to as strange wave in literature, propagating roughly at the speed of sound in combustion products. The combined effect of shock induced preheating and viscous heating near walls seem to be responsible for the formation of the complex flameshock interactions observed. A simple thermodynamic analysis applied to the strange wave using the experimentally measured wave speed yield a pressure ratio of $\sim 15$ during its steady propagation; furthermore, an estimate of the total energy losses required to thermodynamically realize such propagation regime revealed that approximately half of the energy released by combustion should be dissipated (i.e. momentum and heat losses). Finally, simultaneous two-direction optical access allows to map the exact location of detonation onset, showing that $78 \%$ of cases exploded in corners, highlighting the role of corner flows and boundary layers in transition to detonation at this scale.
\end{abstract}

\section{Introduction}

To comply with international agreements limiting $\mathrm{CO}_{2}$ emissions, different solutions have been proposed to reduce the share of fossil fuels in electricity generation and transportation (Masson-Delmotte et al., 2018). The increased use of renewables (e.g. wind, solar) requires efficient/lasting storage solutions due to its intermittent nature. Among them battery banks or electrolyzers to produce hydrogen $\left(\mathrm{H}_{2}\right) . \mathrm{H}_{2}$ has the potential to be a key player in enabling a sustainable energy transition. Its widespread use in transportation, for instance, poses risks due to leaks in refueling stations for vehicles (such as the accident in Sandvika, Norway 2019) or in fuel cell/battery enclosures that can lead to strong explosions via deflagration-to-detonation transition (DDT). Note that the typical gaps in these compartments are of the order of millimeters, therefore understanding of DDT at this scale is needed to mitigate the aforementioned hazards through improved, research-based design.

Towards that goal, a canonical configuration, namely a 1-m long, optically accessible, square cross-section channel $(10 \mathrm{~mm} \times 10 \mathrm{~mm})$ was built to study all stages of DDT: from ignition, flame propagation and acceleration, to transition to detonation. However, here, we are only interested in characterizing the dynamics before and during detonation onset (i.e. formation of flame-shock complexes) and revealing their three-dimensional structure. Studying this stage is essential to understand how to promote or mitigate DDT in narrow channels. Previous numerical and experimental work on flame-shock interaction (Khokhlov et al., 1999; Thomas et al., 2001) have identified interesting structures that lead to DDT; Yanez \& Kuznetsov (2016) also reported similar observations but using narrow channels in which very wrinkled funnel-shape flames trail behind planar shocks. These quasi-steady flame-shock complexes propagate at speeds on the order of the speed of sound in combustion products; the authors called this regime strange wave.

In the present work, two channels allowing one- and two-direction simultaneous schlieren visualization, respectively, are used to study the formation of strange waves.

\footnotetext{
*Corresponding authors: yves.ballossier@ensma.fr

**Principal corresponding authors: florent.virot@ensma.fr; josue.melguizo-gavilanes@cnrs.pprime.fr ORCID(s): 0000-0001-7511-2910 (Y. Ballossier)
} 


\section{Experiments}

\subsection{Apparatus and procedure}

The experimental apparatus was designed to allow flexibility regarding optical access. Its total length is $1030 \mathrm{~mm}$; $91 \%$ of which is optically accessible. To switch from one-direction (1-DV) to two-direction visualization (2-DV) only the center piece of the channel needs being interchanged (see cross sectional views in Fig. 1). In both configurations, $15-\mathrm{mm}$ thick polycarbonate plates are used as windows. Aluminum flanges on top and bottom avoid bending of the polycarbonate during detonation onset and propagation. For the 2-DV configuration, spacers prevent bending on the sides (one of them is visible in Fig. 8 bottom view). None of the tests performed showed signs of deformation. Structural integrity is ensured with bolts screwed on the aluminum in a "sandwich" configuration.

The experiments were conducted as follows: (i) the channel was vacuumed to an absolute pressure below $100 \mathrm{~Pa}$ (controlled by a MKS 220DA pressure sensor); (ii) a plastic cap, held on a servo motor's arm, sealed the open end of the channel; (iii) subsequently, the reactive mixture (stoichiometric $\mathrm{H}_{2}-\mathrm{O}_{2}$ ) was fed into the channel until it reached atmospheric pressure. At this point, the plastic cap was automatically removed by the servo motor; (iv) one second later the mixture is ignited $\left(E_{\text {ign }}<1 \mathrm{~mJ}\right)$ at the closed end by the electric arc that forms between the electrodes. This procedure ensured adequate control of the mixture composition $(\Phi=1 \pm 0.01)$. The waiting time between filling and ignition was also carefully controlled; $15 \mathrm{~s}$ were found sufficient to avoid any impact of initial flow unsteadiness on early stages of flame propagation (Ballossier et al., 2021). After each test, the channel was vacuumed for a few minutes to remove any visible condensation from burned gases.

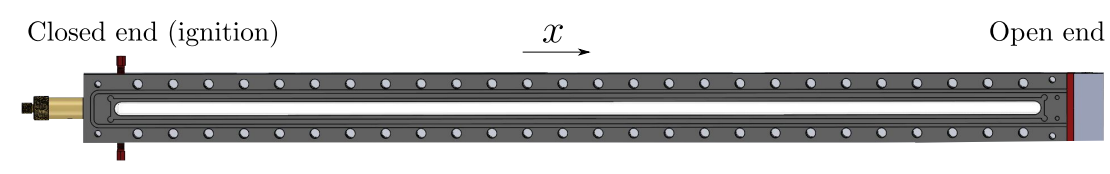

Polycarbonate optical access

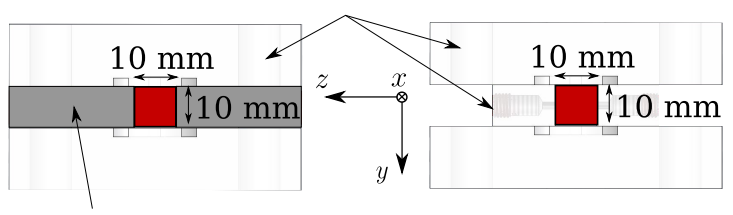

Center piece aluminum part

Figure 1: Schematic of narrow channel (top) and cross-sections for 1-DV (bottom left) and 2-DV (bottom right). Red squares indicate effective cross sections of the channel; white spaces show optically accessible regions.

\subsection{Flow visualization}

Schlieren visualization (SV) is performed on both channels. Two sets of cameras were used for 2-DV visualization:

(i) Two black and white Photron FASTCAM SA-Z both set at 140k frame per second (fps) but with different exposures times, $158 \mathrm{~ns}$ and $248 \mathrm{~ns}$; (ii) Shimadzu HPVX camera, with acquisition rates of 1 million (500 ns exposure) to 10 million fps (50 ns exposure), together with a Shimadzu HPVX-2 camera set at 1 million fps (500 ns exposure).

Different light sources were utilized: two 100-mm diameter collimated LED, and a mercury light, coupled with a collimating mirror also of $100 \mathrm{~mm}$ in diameter. While the collimated LED offers compactness, the use of a mercury light provides a smaller focal point that results in a larger range of observable gradients (the side view in Fig. 8 is the only case using the latter). For the remaining cases shown, the collimated LED set-up is used with a razor blade cutting off about $50 \%$ of the light to highlight gradients in the direction of flame propagation. Our schlieren set-up allows to visualize a section up to $100 \mathrm{~mm}$ with resolutions varying from $178 \mu \mathrm{m}$ to $238 \mu \mathrm{m}$ per pixel; 2-DV is performed on the same section when done simultaneously. Figure 2 shows a simplified schematic of the global optical set-up. For 1-DV, only the optical path of the side view with the HPVX-2 is used. To trigger the high speed camera(s), a BPW34 photo-diode, placed before the section of interest detects the arrival of the flame. 


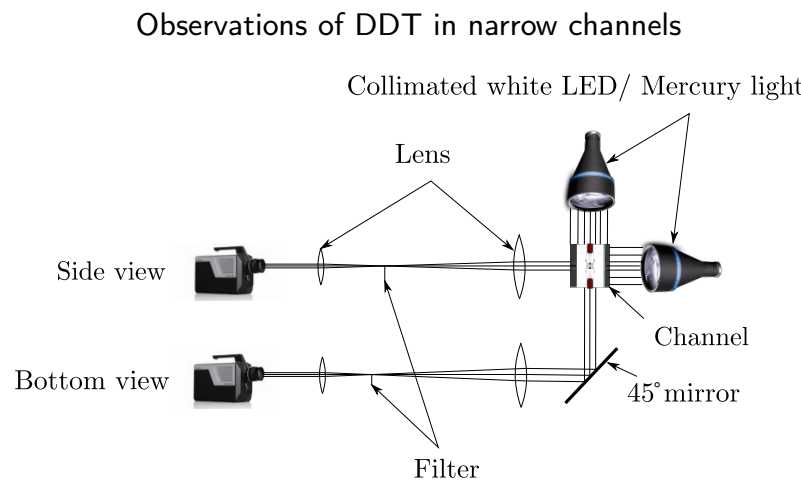

Figure 2: Schematic of global optical set-up for 1-DV and 2-DV.

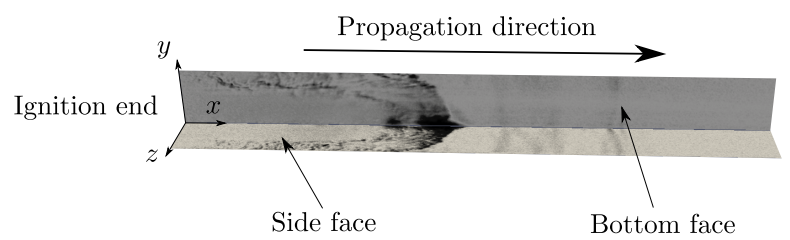

Figure 3: Schematic of the visualization directions. "Side" and "Bottom" faces refer to the camera position.

\section{Results and discussion}

For the sake of clarity in the presentation of the results that follow, Fig. 3 presents a schematic of the visualization orientations. Note that "Bottom" and "Side" faces refer to the relative position of the cameras; the actual position of the bottom face along the $z$-axis (or side face along $y$-axis) is arbitrary, as SV only provides integrated information along the line of sight. The chosen representation is convenient when interesting dynamics occur in the lower right corner as seen from the ignition end. Post-processing typically consists of histogram and resolution matching to obtain similar gray-scales in the figures; a frame-by-frame subtraction of the average field obtained over the sequence collected; followed by a color inversion. The aforementioned steps were automated via a Python script, and the leftover noise ahead of the reacting fronts was manually removed. The final images were carefully checked and compared with the original videos afterwards to ensure that no artifacts were mistakenly produced as a result of our post-processing scheme. From the images collected, positions were extracted to plot in $x-t$ diagrams below the corresponding cases; symbols (circles) are used to represent discrete data. The position data was then fitted automatically to either a first or second order polynomial depending on the $R^{2}$-value obtained, and subsequently differentiated to extract velocities and accelerations when possible. The methodology described yields a $\pm 3.7 \%$ and $\pm 5 \%$ in the reported velocities for tests performed using a resolution of $178 \mu \mathrm{m} /$ pixel and $238 \mu \mathrm{m} /$ pixel, respectively. Finally, the times on the $x-t$ diagrams are relative to photo-diode detection, and do not correspond to the arbitrary reference used in the corresponding images. A total of 54 tests were performed of which 18 used 1-DV and 36 used 2-DV.

\subsection{Strange wave dynamics}

\subsubsection{Early stages and formation}

Figure 4 top shows the structure observed during formation of the strange wave using 1-DV. Time $t_{\text {ref }}$ is arbitrary, and simply represents the first instance in the sequence. The tip of a very corrugated flame (A) is seen to propagate from left to right at $t=t_{\text {ref. }}$. Expansion of burned products and the acceleration of the flame itself, generate perturbations within the fresh but shocked mixture ahead of the flame (weak (B) and strong (C) pressures waves are visible in this frame). At $t=t_{\text {ref }}+27.5 \mu \mathrm{s}$, strengthening of flame acceleration and successive preheating from precursor shocks/pressure waves result in new, stronger shocks, forming closer to the flame (B). The flame starts to show a rather symmetric shape, preferentially burning near walls, resembling a funnel-shape (D) presumably filled with unburned mixture, finally, the flame catches up with the shock at $t=t_{\text {ref }}+55 \mu \mathrm{s}$ (not shown as it is outside of the viewing window). Note that an explosion emanating somewhere on the bottom face is observed, remnants of which are visi- 

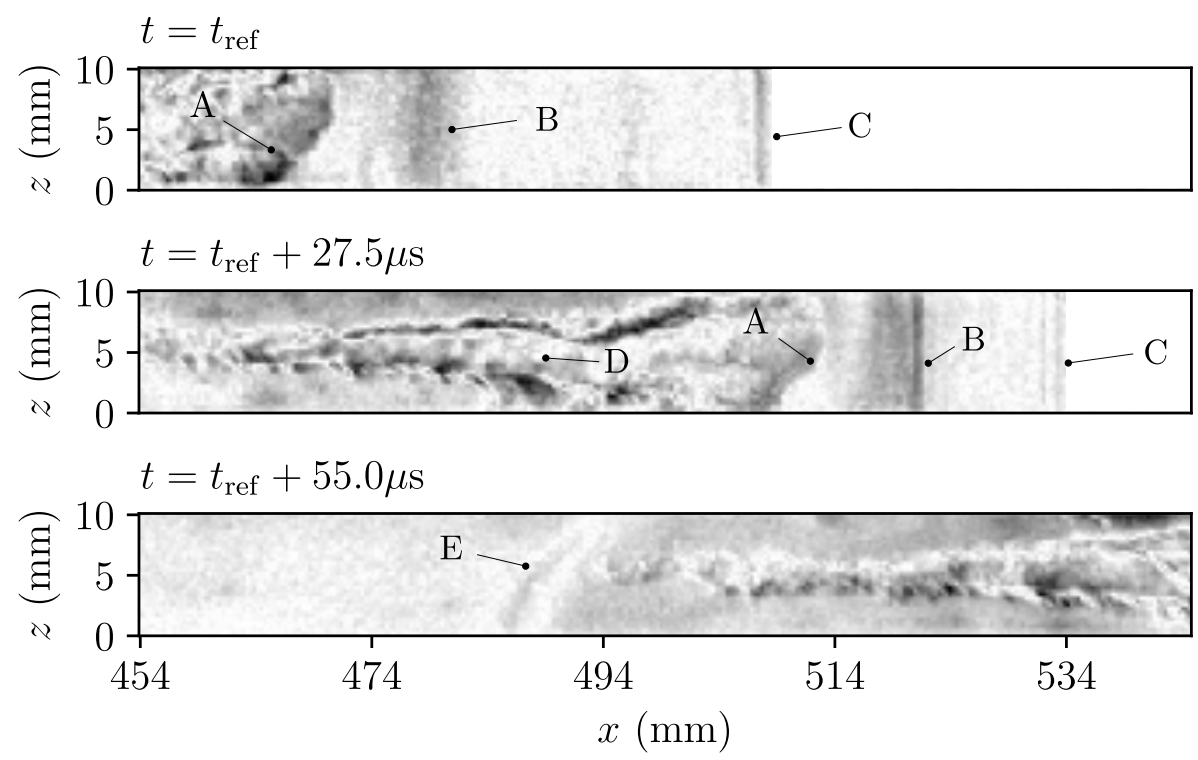

\section{○ Shock merging - $\quad$ S Shock merging $+(\mathrm{C}) \quad$ O Flame $(\mathrm{A})$}
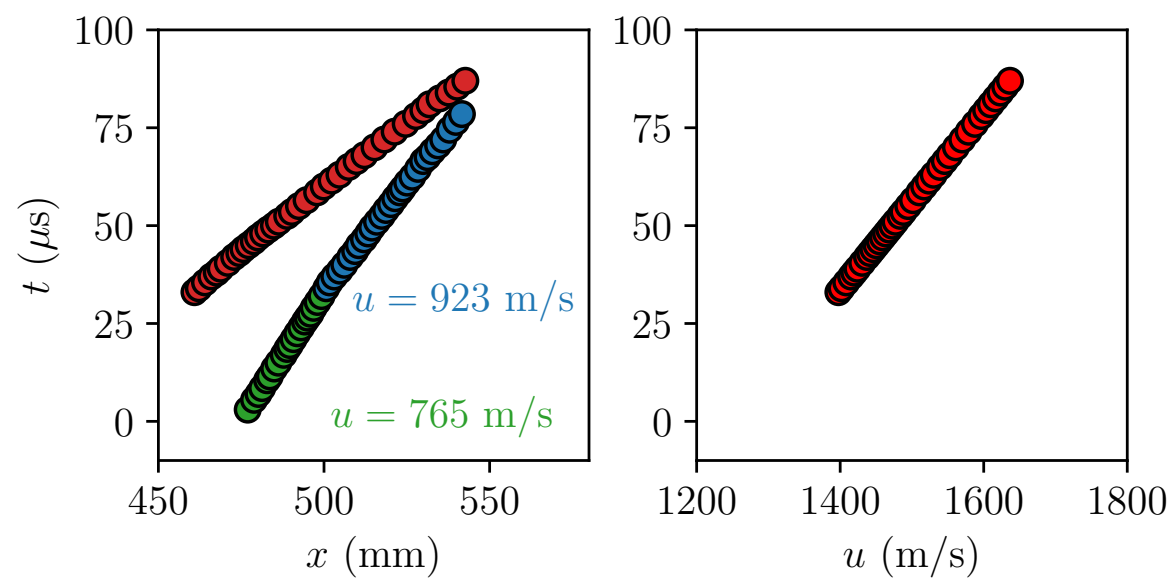

Figure 4: 1-DV of symmetric formation of strange wave; section 454 to $544 \mathrm{~mm}$ from ignition; conditions: stoichiometric $\mathrm{H}_{2}-\mathrm{O}_{2}$ at $p_{0}=100 \mathrm{kPa}$, and $T_{0}=290 \mathrm{~K}$. A: corrugated front; B: weak pressure waves; C: precursor shock; D: funnel-shaped flame surface; E: left moving discontinuity.

ble as a discontinuity traveling left (E) whose propagation speed decreases from $2800 \mathrm{~m} / \mathrm{s}$ to $2300 \mathrm{~m} / \mathrm{s}$. Not enough information is available to tell whether this local explosion lead to DDT. Front speeds computed from the images (see Fig. 4 bottom) increase from $1400-1600 \mathrm{~m} / \mathrm{s}$ over $50 \mu \mathrm{s}$ for the corrugated flame, and from around $700-1000 \mathrm{~m} / \mathrm{s}$ over $80 \mu \mathrm{s}$ for the precursor shock as seen in the $x-t$ diagram. The change of slope associated with the precursor shock (C) around $40 \mu \mathrm{s}$ is due to a trailing shock (not shown) that catches up and merges with (C).

Interestingly, in some cases using the same configuration and viewing section, another structure was observed (see Fig. 5 top). In this case no perturbations seem to be visible ahead of the flame, instead, a front propagating within the boundary layer at the bottom wall forms an oblique shock $(\mathrm{F})$. The oblique shock in this case propagates at a similar 

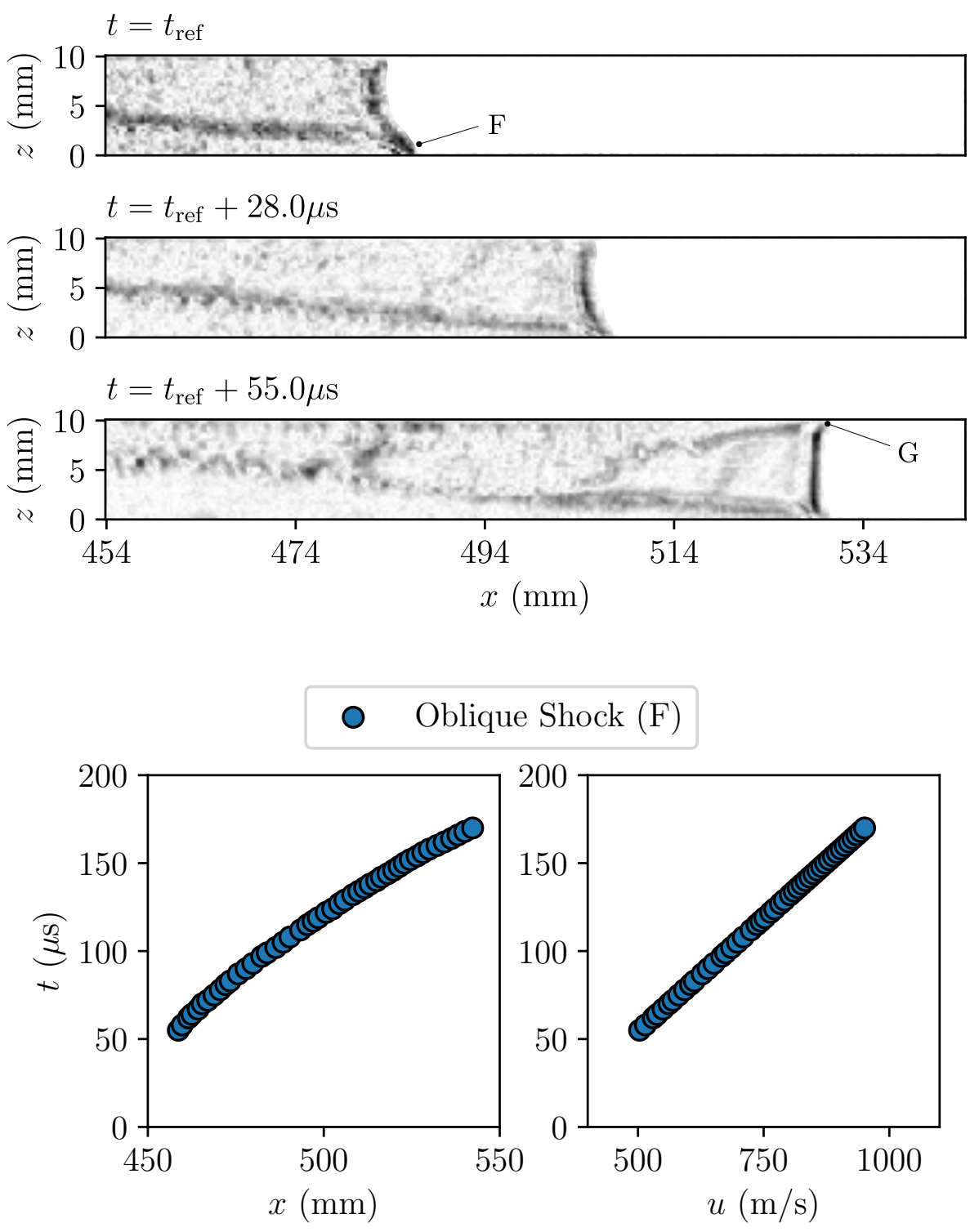

Figure 5: 1-DV of asymmetric formation of strange wave; section 454 to $544 \mathrm{~mm}$ from ignition; conditions: stoichiometric $\mathrm{H}_{2}-\mathrm{O}_{2}$ at $p_{0}=100 \mathrm{kPa}$, and $T_{0}=290 \mathrm{~K}$. F: oblique shock; G: second ignition.

speed (see Fig. 5-bottom) as that reported for the precursor shock in Fig. 4. Upon reflection from the top wall, a second ignition within the boundary layer takes place $(\mathrm{G})$. On the last frame, at $55 \mu \mathrm{s}$, a symmetric shape starts to form. This shape is very similar to that observed experimentally by Thomas et al. (2001), numerically by Khokhlov et al. (1999), and Oran et al. (2002) when they studied shock-flame interactions.

Figure 6 shows 2-DV during formation of the strange wave. Note that in this channel this stage of DDT occurred in a different section, $281 \mathrm{~mm} \leq x \leq 353 \mathrm{~mm}$, instead of $454 \mathrm{~mm} \leq x \leq 544 \mathrm{~mm}$. At $t=t_{\text {ref }}$, this configuration shows a similar structure as in Fig. 4 preceded by a succession of pressure waves. The main difference lies on the fact that the corrugated flame does not catch up with the perturbations, instead the mixture seems to ignite within the boundary layer, likely due to a flame-boundary layer interaction, resulting in a front that propagates near the wall (similar to that seen in Fig. 5). Simultaneous SV allows to pinpoint the exact location on the channel cross-section where ignition 

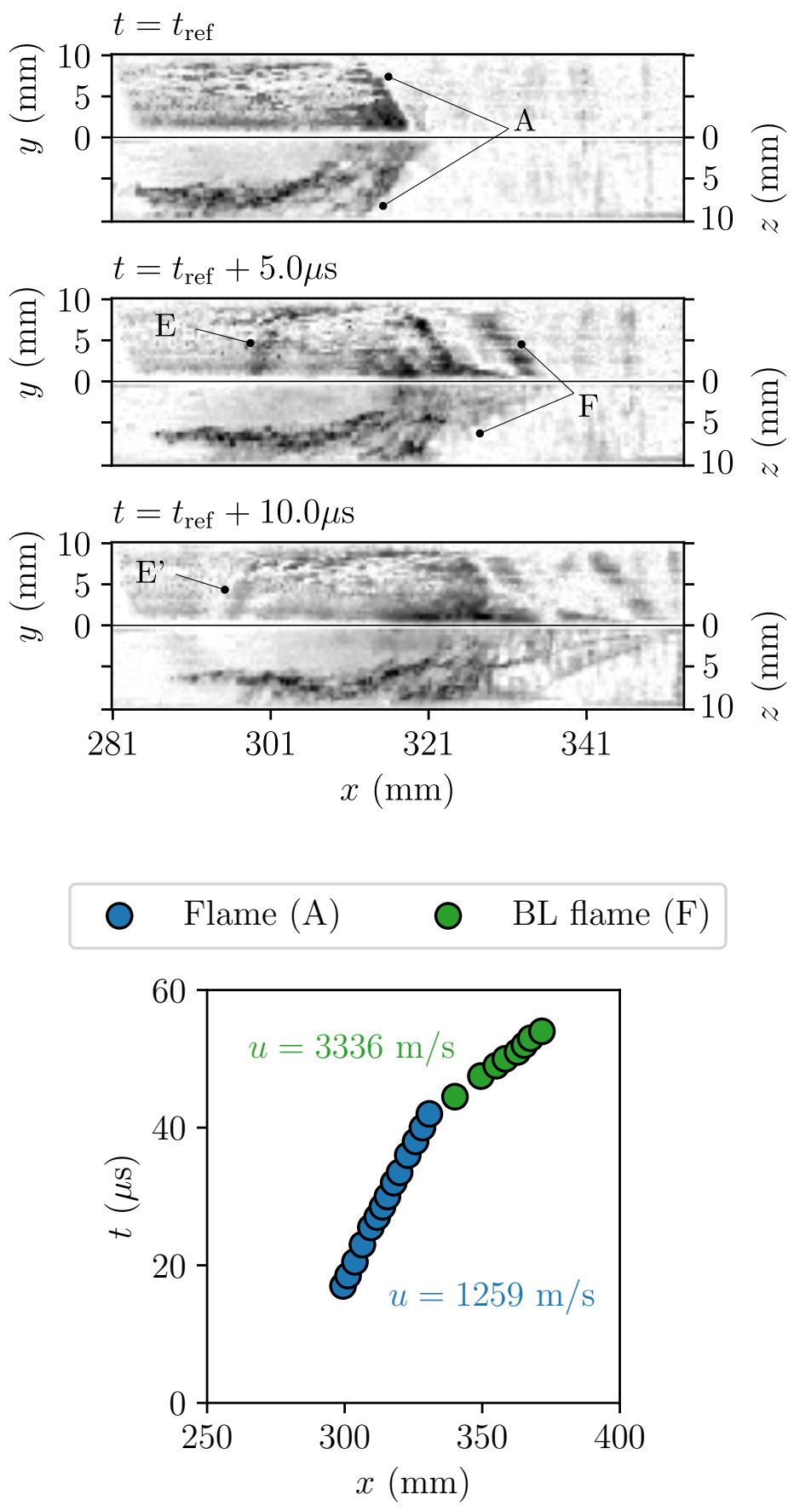

Figure 6: Simultaneous 2-DV of the formation of strange wave; section 281 to $353 \mathrm{~mm}$ from ignition; conditions: stoichiometric $\mathrm{H}_{2}-\mathrm{O}_{2}$ at $p_{0}=100 \mathrm{kPa}$, and $T_{0}=290 \mathrm{~K}$. Background substracted to highlight important flow features. A: corrugated front; F: boundary layer flame - oblique shock; E: left moving discontinuity.

takes place (bottom right corner looking from the ignition end). At $t=t_{\text {ref }}+5 \mu \mathrm{s}$, the front in the boundary layer (F) propagates faster than the corrugated flame $(\sim 3300 \mathrm{~m} / \mathrm{s})$. In the side view, at $t=t_{\text {ref }}+10 \mu \mathrm{s}$, another fast front starts to propagate within the boundary layer at the lower edge of this frame. The ignition of this front seems to be the result 
of an oblique shock-boundary layer interaction. However, based on a survey of all the results collected, ignition within the boundary layers arise more frequently due to flame-boundary layer interactions. The exact reason of spontaneous ignition within the corner is not clear, additional tests and analysis are currently being carried out to rule out uncertainties concerning manufacturing imperfections; more details are provided in section 3.2. The DNS of Dziemińska \& Hayashi (2013) and LES of Zhao et al. (2017) present a similar scenario of DDT, but in our case, the flame-shock complex propagates more than $100 \mathrm{~mm}$ before it actually transits to detonation. A discontinuity (E), similar to that seen in Fig. 4 at $t=t_{\text {ref }}+55 \mu \mathrm{s}$, is also visible at $t=t_{\text {ref }}+5 \mu \mathrm{s}$ and $t=t_{\text {ref }}+10 \mu \mathrm{s}$. The overall dynamics obtained resembles that for 1-DV.
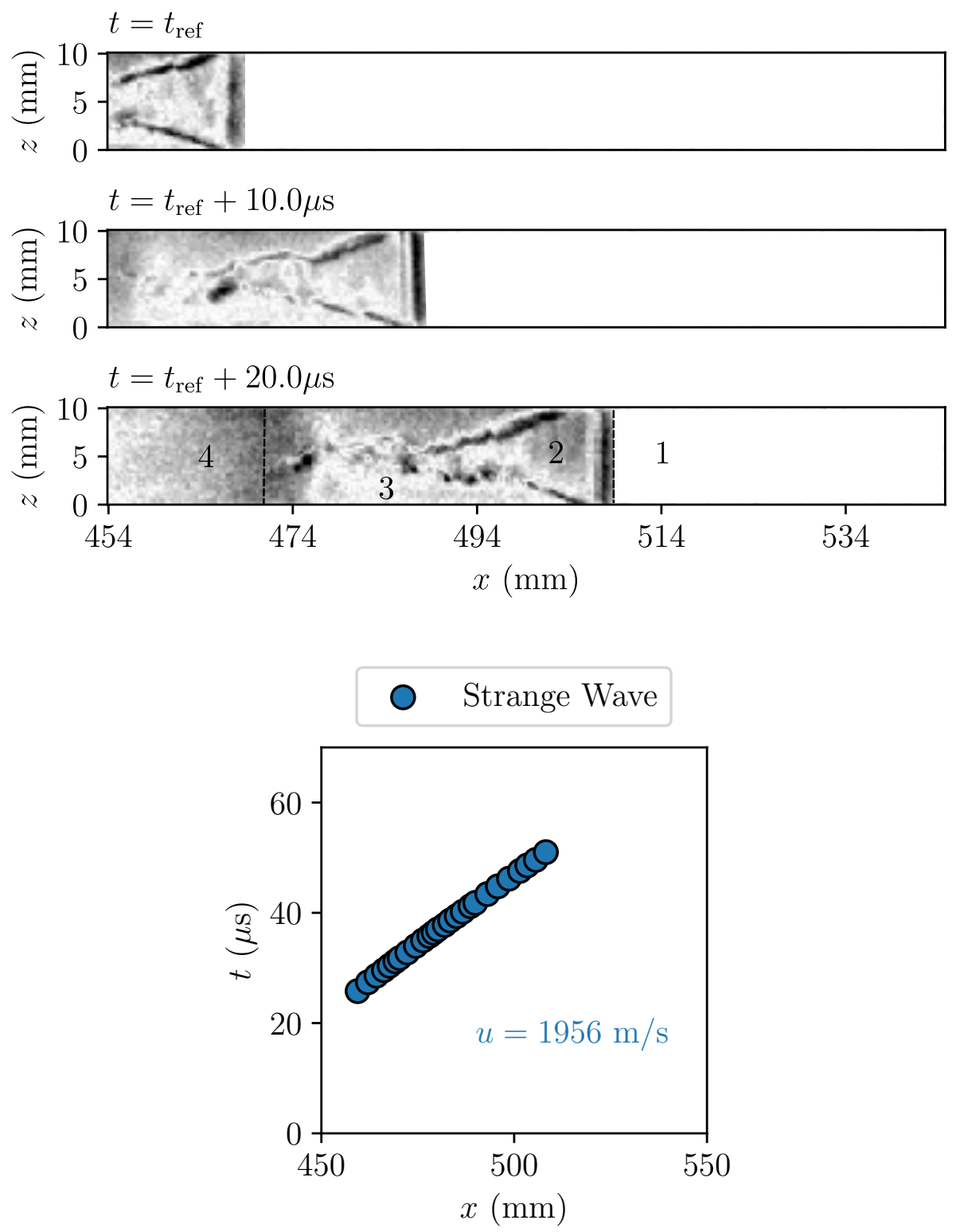

Figure 7: 1-DV of strange wave; section 454 to $544 \mathrm{~mm}$ from ignition; conditions: stoichiometric $\mathrm{H}_{2}-\mathrm{O}_{2}$ at $p_{0}=100 \mathrm{kPa}$, and $T_{0}=290 \mathrm{~K}$. 


\subsubsection{Quasi-steady propagation}

The structure of the strange wave is visible in Fig. 7 top. It is very similar to that observed by Yanez \& Kuznetsov (2016), except that in our case it propagates at a speed $\sim 29 \%$ higher $\left(u_{S W} \sim 1940 \mathrm{~m} / \mathrm{s}\right)$. As mentioned above, it is composed of a flat shock with a very wrinkled funnel-shaped flame surface responsible for sustaining the very high burning rates observed. The 2-DV shown in Fig. 8, reveals additional information. At $t=t_{\text {ref }}$, the Bottom view and Side view exhibit very different shapes. From the side, a similar shape as that observed in the first configuration, with a planar shock ahead of a funnel-shaped flame, whereas from the bottom, only a planar shock is visible, just before the white spacer. Even though this flame-shock complex is supersonic with respect to reactants ahead, it is not the quasi-steady strange wave observed by Yanez \& Kuznetsov (2016). The planar shock propagates around $1250 \mathrm{~m} / \mathrm{s}$ and it is quickly overtaken by the funnel-shaped flame. When they merge near the center, at $t=t_{\text {ref }}+18 \mu \mathrm{s}$, the complex accelerates to $\sim 1800-1900 \mathrm{~m} / \mathrm{s}$ and starts to flatten, sometimes staying curved in one corner until detonation onset. The Bottom view at $t=t_{\text {ref }}+18 \mu \mathrm{s}$ is evidence of the convoluted three-dimensional structure that forms when both oblique flame-shock complexes merge.

\subsubsection{Thermodynamic analysis}

The metastable propagation velocities shown in Fig. 7 motivate the applicability of a simple thermodynamic analysis to investigate these structures. Yanez \& Kuznetsov (2016) first proposed this approach and showed the existence of a solution in the pressure-specific volume diagram $(p-v)$ that is different but close to that of a steadily propagating detonation. In their work the authors took the structure shown in Fig. 7 and identified four distinct states (annotated in the figure) and used the Rankine-Hugoniot jump conditions to find a solution for the wave speed, $u_{S W}$, that would close the resulting system of equations. The following assumptions were made in their analysis:

(i) $1 \rightarrow 2$ : fresh mixture is adiabatically compressed by a shock raising its pressure and temperature whose Rayleigh line (RL) and Hugoniot curve (HC) are:

$$
\mathrm{RL}:\left(\frac{u_{S W}}{v_{1}}\right)^{2}=\frac{\left(p_{2}-p_{1}\right)}{\left(v_{1}-v_{2}\right)} ; \quad \mathrm{HC}: \frac{\gamma_{2}}{\gamma_{2}-1} p_{1} v_{1}-\frac{\gamma_{2}}{\gamma_{2}-1} p_{2} v_{2}+\frac{1}{2}\left(v_{1}+v_{2}\right)\left(p_{2}-p_{1}\right)=0
$$

(ii) $2 \rightarrow$ 3: quasi isobaric combustion;

$$
\mathrm{RL}:\left(\frac{S_{L}}{v_{2}}\right)^{2}=\frac{\left(p_{3}-p_{2}\right)}{\left(v_{2}-v_{3}\right)} ; \quad \mathrm{HC}: \frac{\gamma_{2}}{\gamma_{2}-1} p_{2} v_{2}-\left(\frac{\gamma_{3}}{\gamma_{3}-1} p_{3} v_{3}+Q\right)+\frac{1}{2}\left(v_{2}+v_{3}\right)\left(p_{3}-p_{2}\right)=0
$$

(iii) $3 \rightarrow 4$ : adiabatic expansion (AE);

$$
\mathrm{AE}: p_{4} v_{4}^{\gamma_{3}}=p_{3} v_{3}^{\gamma_{3}} ; \quad \mathrm{RL}:\left(\frac{u_{S W}}{v_{1}}\right)^{2}=\frac{\left(p_{4}-p_{1}\right)}{\left(v_{1}-v_{4}\right)}
$$

The RL in (iii) enforces the tangency condition that allows to find a solution to the system. In Eqns. (1), (2) and (3), $p, v$ are the pressure in $\mathrm{Pa}$ and specific volume in $\mathrm{m}^{3} / \mathrm{kg} ; \gamma, S_{L}$, and $Q$ are the ratio of specific heats, laminar burning velocity and chemical heat release, respectively; the latter three are case specific parameters that depend on the mixture considered. Finally, the subscripts denote the states where the properties are evaluated.

To find appropriate values for $\gamma_{2}, \gamma_{3}$ and $Q$, the Shock and Detonation Toolbox (SDT) (Browne et al., 2008) is used to compute a reacted Hugoniot curve using temperature dependent thermodynamics for a stochiometric $\mathrm{H}_{2}-\mathrm{O}_{2}$ at $p_{0}=100 \mathrm{kPa}$ and $T_{0}=290 \mathrm{~K}$. An almost perfect match was obtained to expand the products from the von Neumann $(\mathrm{vN})$ to the Chapman-Jouguet (CJ) state using $\gamma_{2}=\gamma_{v N}=1.32, \gamma_{3}=\gamma_{C J}=1.21$ and $Q=8 \mathrm{MJ} / \mathrm{kg}$ for HC in Eqn. (2). $S_{L}$ can be computed with Cantera (Goodwin et al., 2009) for the thermodynamic state given by the intersection of RL and $\mathrm{HC}$ in Eq. (1).

Figure 9-top shows the solution obtained by Yanez \& Kuznetsov (2016) which yields a strange wave propagation 

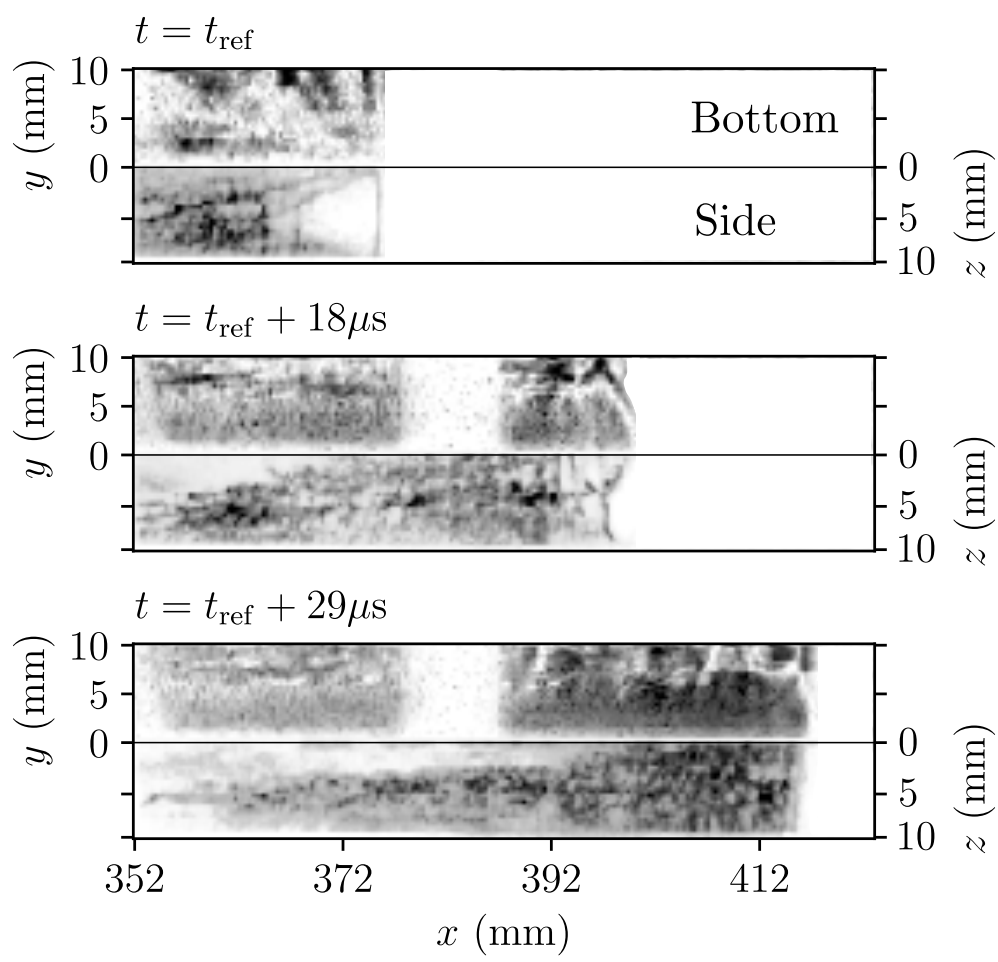

\section{○ Flame merging - $\quad$ F $\quad$ Flame merging +}

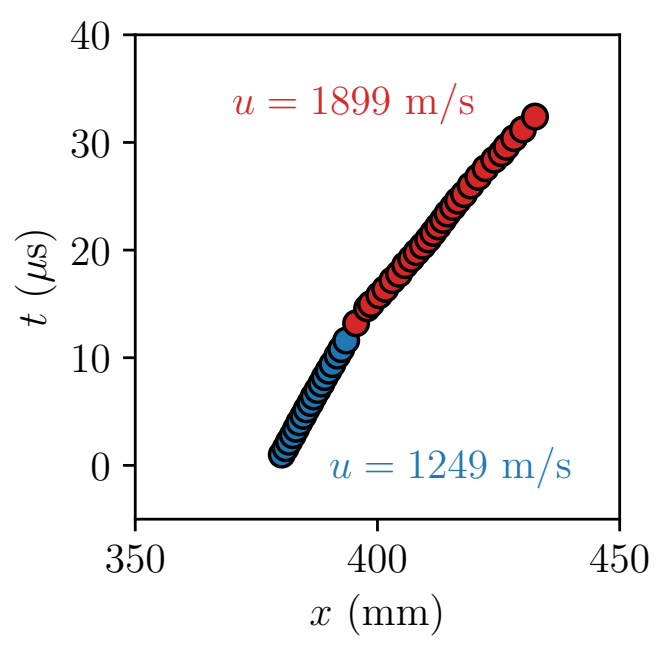

Figure 8: Simultaneous 2-DV of the formation of strange wave; section 352 to $423 \mathrm{~mm}$ from ignition; conditions: stoichiometric $\mathrm{H}_{2}-\mathrm{O}_{2}$ at $p_{0}=100 \mathrm{kPa}$, and $T_{0}=290 \mathrm{~K}$.

speed of $u_{S W}=2750 \mathrm{~m} / \mathrm{s}, 3 \%$ lower than the ideal CJ velocity, $u_{C J}=2835 \mathrm{~m} / \mathrm{s}$. Note that this value is significantly higher than the experimental velocity measurements and is due to the absence of any dissipation mechanisms in the analysis performed. To compute a first order approximation of the energy losses required to realize the experimentally observed velocities, we repeated the exercise taking the wave speed measured in Fig 7 as an input, and using $Q$ as a 


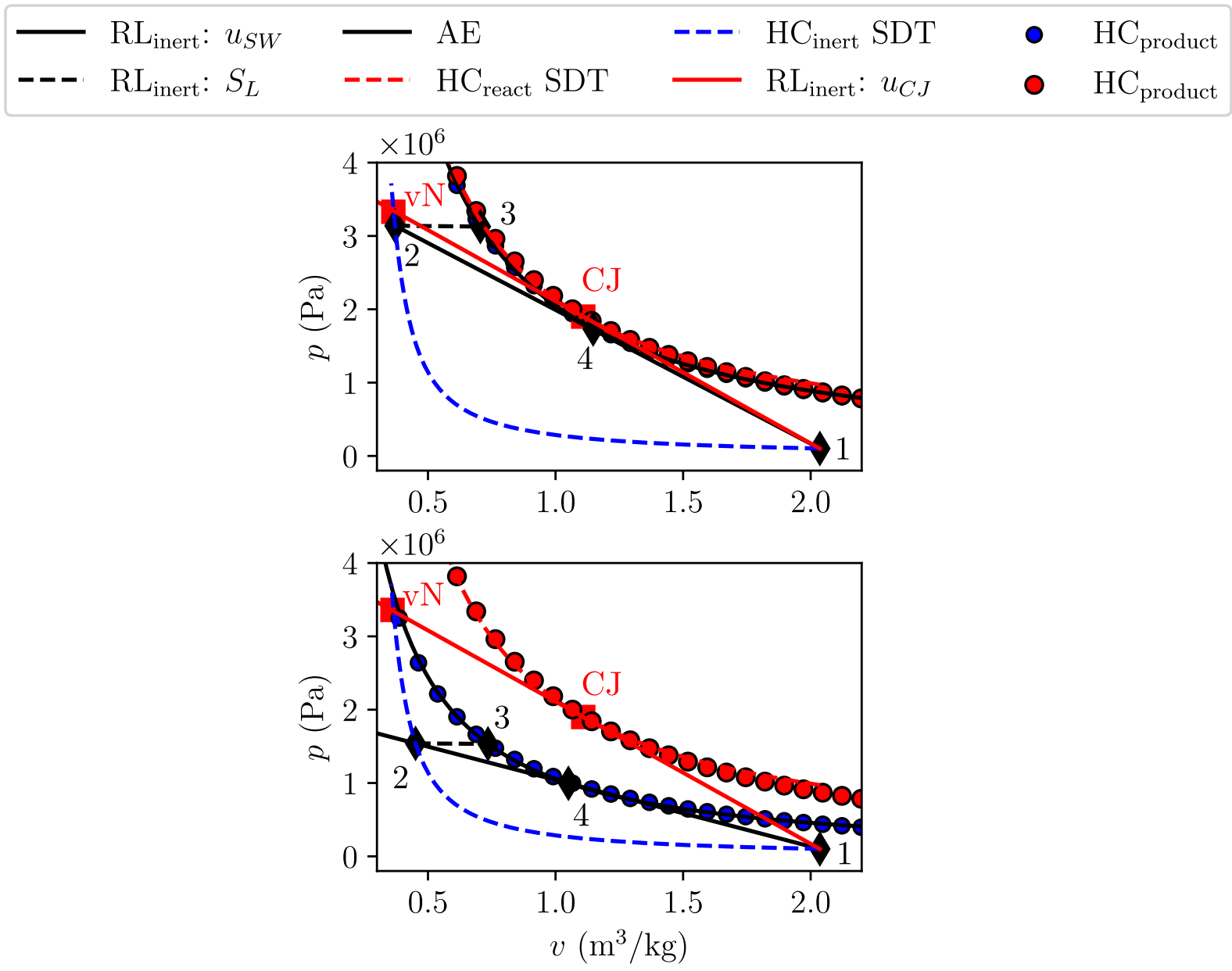

Figure 9: $P-v$ diagrams obtained for strange wave analysis. Top: solution of Yanez \& Kuznetsov (2016); bottom: solution using experimental wave propagation velocity, $u_{\mathrm{SW}}$, as an input.

free parameter. A value of $Q=3.9 \mathrm{MJ} / \mathrm{kg}$ satisfies the system of equations (see Fig. 9-bottom) suggesting that about half of the chemical heat release should be quickly dissipated in the form of heat and momentum losses; however, a simple analysis of the characteristic time scales present in our configuration revealed that the heat dissipation time over half the channel height $(5 \mathrm{~mm})$ is 7 times longer than the transit time of the wave over its average length $(31 \mathrm{~mm})$, suggesting that heat losses should contribute little to the overall losses. The intersection of the RL with the unreacted Hugoniot indicates that the associated pressure ratio, $p_{2} / p_{1} \sim 15$, is less than half that shown in Fig. 9-top. We note that the velocity deficits observed for the strange waves reported here $\left(u_{S W} / u_{C J} \sim 2 / 3\right)$ are larger than those that have been reported and analyzed for steadily propagating detonations in channels by Fay (1959); Tsugé (1971); Dove et al. (1974); Zel'dovich et al. (1987) and others. As a limiting case, if across the strange wave the gases were to be expanded directly from state 2 to 4 following the corresponding RL instead of the thermodynamic path 2-3-4, the same final state is obtained.

A simpler, equivalent way of estimating the losses is by taking directly the expression derived from CJ theory assuming a single- $\gamma$ model (see Fickett \& Davis (2000)) to write $u_{C J}=\sqrt{2\left(\gamma^{2}-1\right) Q}$ and $u_{S W}=\sqrt{2\left(\gamma^{2}-1\right)(Q-\bar{q})}$, where $\bar{q}$ represents the combined effective losses. Taking their ratio and manipulating yields $\bar{q} / Q=1-\left(u_{S W} / u_{C J}\right)^{2}=0.52$. We emphasize that the result above is mainly of qualitative nature, and that further refinements to the thermodynamic model can be achieved by revisiting the assumptions of quasi-isobaric combustion and adiabatic expansion of the gases between states 2-3 and 3-4, respectively, or better, by extending the analysis to a 1-D description of the flow including 
friction and heat losses.

\subsection{Detonation onset}

Transition to detonation systematically occurs following the strange wave structure described above for 1-DV cases. Figure 10 shows the results obtained using the 1-DV configuration. An ignition kernel develops at the bottom wall at $t=t_{\text {ref }}$. The initially planar shock becomes curved, as the shock generated from the explosion travels towards the top wall, consuming the fresh shocked mixture present downstream of it. Finally, the frame at $t=t_{\text {ref }}+3.2 \mu \mathrm{s}$, shows a quasi-planar shock of an incipient detonation. Run-up distances computed from the images (i.e. DDT location) lied between $38-67 \%$ of the total length of the channel (sample size: 14 tests). The high temporal resolution used allowed us to resolve the detonation onset in great detail ( 5 million fps).

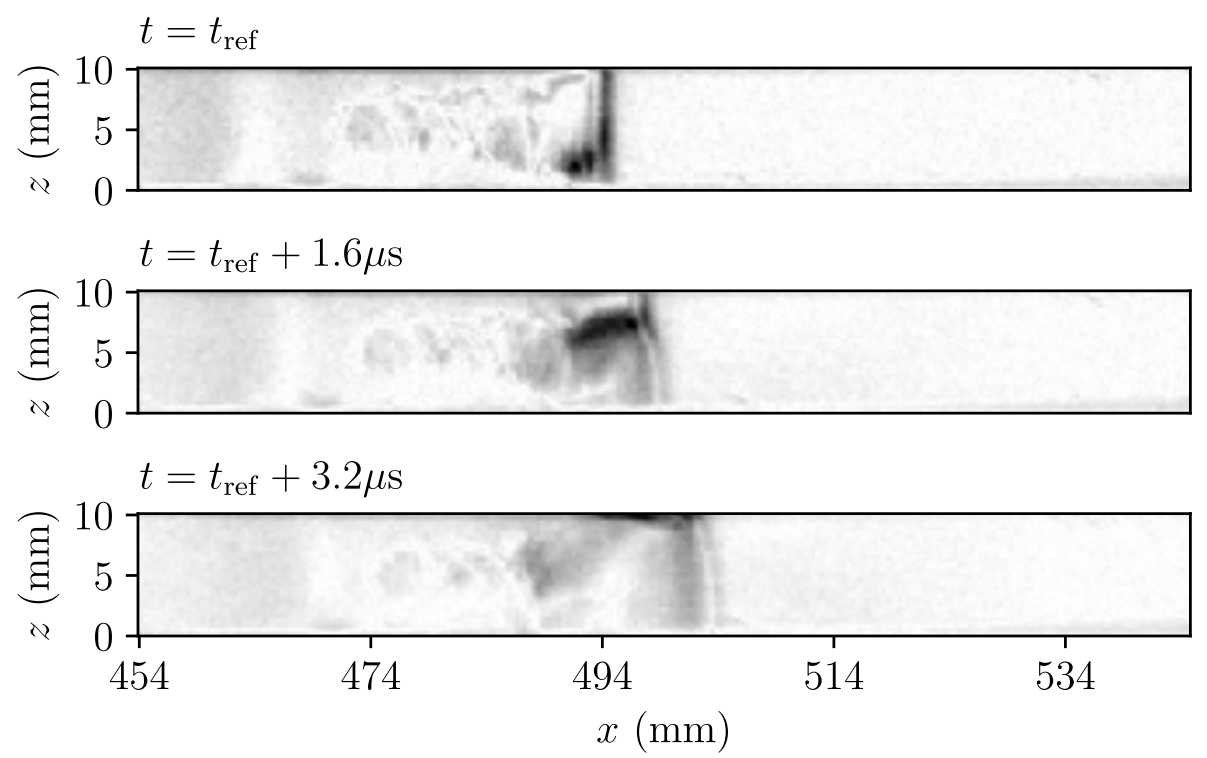

\section{○ DDT onset - $\quad$ D DDT onset +}

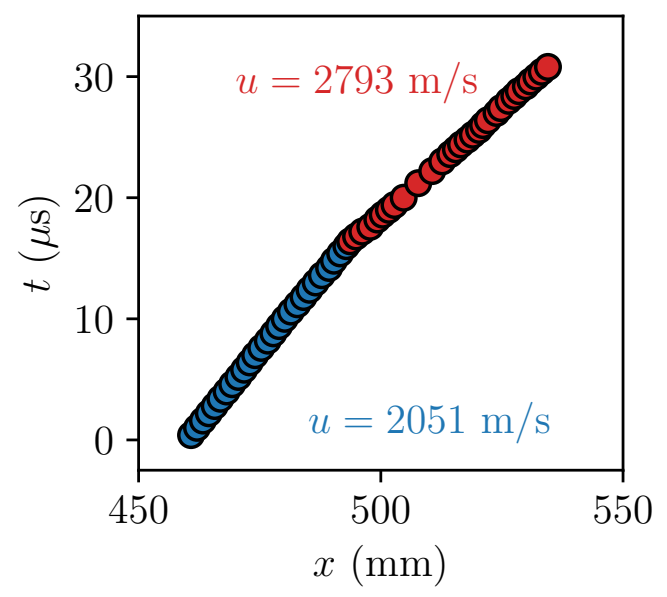

Figure 10: 1-DV of transition to detonation; section: 454 to $544 \mathrm{~mm}$ from ignition; conditions: stoichiometric $\mathrm{H}_{2}-\mathrm{O}_{2}$ at $p_{0}=100 \mathrm{kPa}$, and $T_{0}=290 \mathrm{~K}$. 

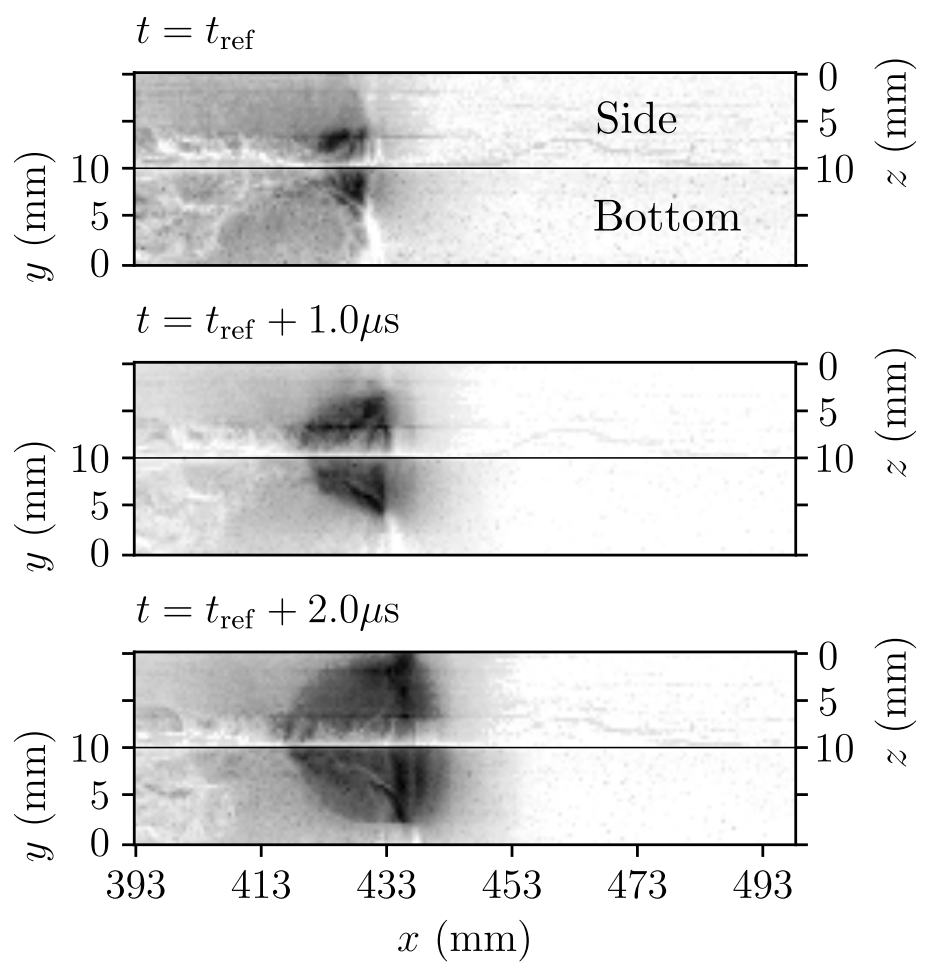

\section{○ DDT onset - $\quad$ D DT onset +}

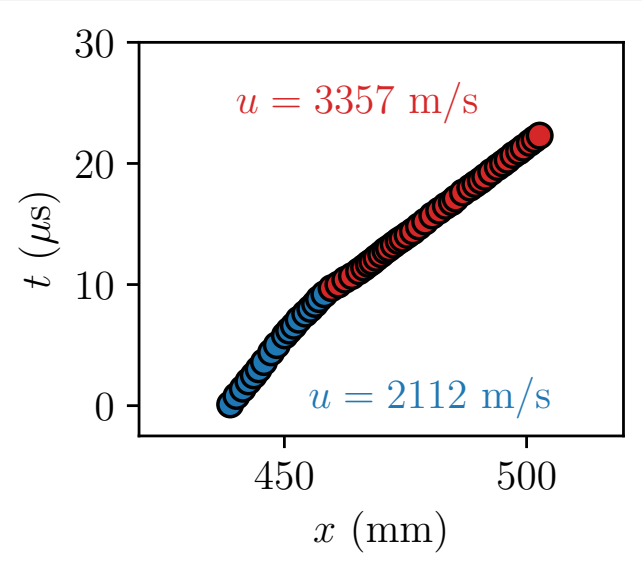

Figure 11: Simultaneous 2-DV of transition to detonation; section: 393 to $498 \mathrm{~mm}$ from ignition, rotated; conditions: stoichiometric $\mathrm{H}_{2}-\mathrm{O}_{2}$ at $p_{0}=100 \mathrm{kPa}$, and $T_{0}=290 \mathrm{~K}$.

Figure 11 shows the results obtained with 2-DV configuration, following the flattening of the complex described in Fig. 8, whose propagation speed is $\sim 2000 \mathrm{~m} / \mathrm{s}$, a strong explosion takes place. Similarly to 1-DV in Fig. 10, when the explosion reaches the opposite walls it reflects as a transverse wave. Note that to be consistent with the schematic in Fig. 3, the planes $x-y$ and $x-z$ were translated, and subsequently rotated over the $x$-axis; the DDT point is actually in the top left corner. Run-up distances lied between $42-47 \%$ of total length (sample size: 20 tests). While wave velocities before detonation onset (DDT onset - ) are of the same order $(u \sim 1900-2000 \mathrm{~m} / \mathrm{s}$ ), wave velocities after detonation onset (DDT onset + ) show significant differences $(700 \mathrm{~m} / \mathrm{s})$ despite the similarities between both con- 
Table 1

Summary of results specifying number of occurrences and structures observed per visualization type. Total sample size: 54 tests.

\begin{tabular}{l|ccc|cc}
\hline \multirow{2}{*}{ Wave type } & \multicolumn{3}{|c|}{ 1-DV } & \multicolumn{2}{c}{ 2-DV } \\
& Sample size & $u(\mathrm{~m} / \mathrm{s})$ & Sample size & $u(\mathrm{~m} / \mathrm{s})$ \\
\hline Symmetric & 4 & 1400 & 9 & 1300 \\
\hline Asymmetric & 3 & $700-2000$ & 0 & None \\
\hline Funnel-shaped & 5 & 1940 & 7 & $1200-2000$ \\
\hline DDT onset - & 6 & 1900 & 20 & 2000 \\
DDT onset + & \multicolumn{3}{|c|}{ 1-DV } & 3300 & \multicolumn{2}{c}{ 2-DV } \\
\hline Parameter & Sample size & $x_{\text {DDT }} / L$ & Sample size & $x_{\text {DDT }} / L$ \\
\hline Run-up distance & 14 & $38-67 \%$ & 20 & $42-47 \%$ \\
\hline
\end{tabular}

figurations. A closer look at our data revealed transients in which velocities reach $u \sim 3100-3500 \mathrm{~m} / \mathrm{s}$, corresponding to overdriven detonations, before relaxing to $u \sim 2760 \mathrm{~m} / \mathrm{s}\left(u / u_{C J} \sim 0.97\right)$. Velocity deficits for steady detonation propagation in narrow channels have been reported in the past, and found to be as large as 10-15\% (Zel'dovich et al., 1987). Correlations between detonation cell widths, $\lambda$, and their velocity deficits can be found in Murray (2008), for example, with larger deficits expected as $\lambda$ approaches the hydraulic diameter of the channel/tube in which the detonation is propagating. For our conditions the channel height-to-cell width ratio is around 10, suggesting that the expected deficits should be small; the $3 \%$ measured is therefore consistent with previously reported data. For 1-DV stronger decelerations were observed than those measured for 2-DV; we are currently investigating why this is the case. A potential cause is the momentum losses associated with the wall roughness of the different materials used; the arithmetic mean roughness/height $(\mathrm{Ra})$ for aluminum $(0.3 \mu \mathrm{m})$ is an order of magnitude larger than that of polycarbonate $(0.03 \mu \mathrm{m})$. Using the model presented in section 3.1.3 we estimated the expected pressure ratios during DDT onset + to be of the order of $p_{2} / p_{1} \sim 50$. All detonation onset cases, irrespective of the configuration used, are accompanied by retonation waves that propagate toward the closed end. A hazard associated to their propagation in industrial settings is their interactions with obstacles/infrastructure that may lead to significant impulse levels resulting in material damage. Despite of this, we emphasize that the over-pressures induced at the location of DDT onset are likely higher; we are considering installing a dynamic pressure sensor at the closed end of the channel to quantify the pressure rise upon retonation wave impact.

SV performed on the same section for 1-DV configuration (454-544 mm) include four different structures (Figures 4, 5, 7 and 10); fully developed detonations, as well as slower deflagrations were also observed but not included here. The 2-DV channel, on the other hand, provided repeatable results for the same viewing window, and each figure shows different sections, as specified on the figures' captions. The discrepancies observed between 1-DV and 2-DV cases are due to manufacturing imperfections that were identified on the edges of the windows, and are currently being corrected. As a consequence, the available reactive volume is about $10 \%$ larger, as there are pockets of reactive mixture in small gaps outside the cross section that perturbs the boundary layer and corner flows. This shows the importance of geometry induced perturbations on the stochasticity of DDT; the latter detonation onset scenario may be more representative of the expected evolution during an accidental ignition event; similar observations have been made for obstructed channels by Goodwin et al. (2016); Teodorczyk et al. (2009); Ren et al. (2017). Table 1 summarizes our results. The wave types and velocities reported correspond to mean flame values during early stages and formation of a funnel shape for "Symmetric" (see annotation (A) in Figs. 4 and 6). "Asymmetric" correspond to the same stage but for structures similar to those shown in Fig. 5 (annotation (F)). "Funnel-shaped" is the mean steady velocity of the strange wave for 1-DV, and the merging of oblique fronts for the 2-DV configuration. "DDT onset" recap the mean pre-detonation (-) and post-detonation (+) velocities for both configurations. Run-up distances are also shown.

The extra information gathered from the 2-DV allows us to map the exact location of detonation onset (see Fig. 12). For $78 \%$ of the cases ignition occurs within a corner, and always close to a wall, elucidating the crucial role played 


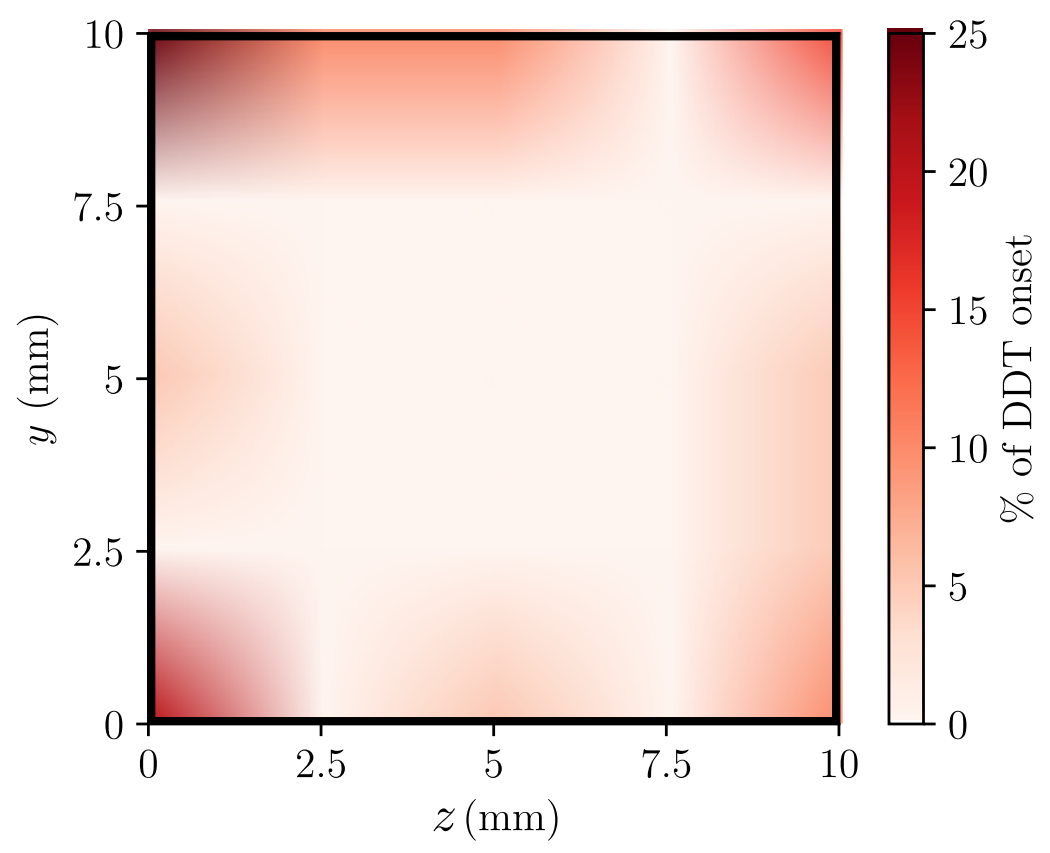

Figure 12: Spatial likelihood of detonation onset on the channel cross-section (looking from the ignition-end) in percentage (sample size: 20 tests). Conditions: stoichiometric $\mathrm{H}_{2}-\mathrm{O}_{2}$ at $p_{0}=100 \mathrm{kPa}$, and $T_{0}=290 \mathrm{~K}$.

by viscous boundary layers and corner flows in detonation onset. Krivosheyev et al. (2020) built a similar map, for highly diluted acetylene-oxygen mixtures, using a different configuration (i.e. a closed, circular 6-m long tube, 60 $\mathrm{mm}$ in diameter). They also observed a high likelihood of DDT close to walls; bear in mind however, that the flow structures and boundary layers that develop behind precursor shocks differ significantly between square and circular cross-sections.

\section{Conclusions}

DDT experiments were conducted using two narrow channels to characterize the three-dimensional structure during detonation onset. One-direction and two-direction simultaneous schlieren visualization was used for that purpose.

The following structures were observed: (i) symmetric funnel-shaped flames; (ii) asymmetric shapes as a result of ignition within boundary layers upon their interactions with the accelerating flame and/or oblique shocks formed by propagating fronts near walls; (iii) quasi-steady propagation of symmetric flame-shock complexes (rather shortlived, for the 2-DV channel); and (iv) detonation onset near the channel's walls. A simple thermodynamic analysis applied to (iii), using the experimentally measured wave speed, allowed to estimate the pressure ratio during its steady propagation $\left(p_{2} / p_{1} \sim 15\right)$ as well as the energy losses required to thermodynamically realize it (approximately half of the energy released by combustion should be dissipated in form of momentum and heat losses). However, a simple estimate and comparison of the characteristic time scale of heat transfer and that associated with the transit of the wave suggests that momentum losses should play a more important role. The main mechanism sustaining the strange wave seems to be a delicate balance between chemical heat release and momentum losses.

Notably, 2-DV allowed us to map the exact location of detonation onset on the channel's cross-section. The highly three-dimensional flows observed, shed light on the importance of understanding the complex structures that develop before DDT, as they may provide important clues regarding mitigation strategies (i.e. quenching of fast flames and suppression of DDT onset). Attenuation of precursors shocks via the use of porous liners may lead to increased velocity 
deficits and suppress altogether the formation of detonations; geometry induced perturbations to the flow, especially at corners, are to be avoided.

Future work will focus on enhancing the quality of the flow visualization and better control of the geometry for 2-DV to allow a finer characterization of the three-dimensional structures observed as an attempt to better understand the role played by corner flows on the DDT dynamics.

\section{Acknowledgements}

The authors gratefully acknowledge l'École Doctorale SIMME, and the contribution of the technical personnel at l'Institut Pprime.

\section{References}

Ballossier, Y., Virot, F., \& Melguizo-Gavilanes, J. (2021). Flame propagation and acceleration in narrow channels: sensitivity to facility specific parameters. Shock Waves,

Browne, S., Ziegler, J., \& Shepherd, J. (2008). Numerical solution methods for shock and detonation jump conditions. GALCIT report FM2006, 6, 90. URL: http://shepherd. caltech.edu/EDL/PublicResources/sdt/.

Dove, J., Scroggie, B., \& Semerjian, H. (1974). Velocity deficits and detonability limits of hydrogen-oxygen detonations. Acta Astronautica, 1, 345-359. doi:https://doi.org/10.1016/0094-5765(74)90102-7.

Dziemińska, E., \& Hayashi, A. K. (2013). Auto-ignition and ddt driven by shock wave-boundary layer interaction in oxyhydrogen mixture. International Journal of Hydrogen Energy, 38, 4185-4193. doi:https : //doi .org/10.1016/j . i jhydene. 2013.01.111.

Fay, J. A. (1959). Two-dimensional gaseous detonations: Velocity deficit. The Physics of Fluids, 2, 283-289. doi:https://doi .org/10.1063/ 1. 1705924.

Fickett, W., \& Davis, W. C. (2000). Detonation: theory and experiment. Courier Corporation.

Goodwin, D. G., Moffat, H. K., \& Speth, R. L. (2009). Cantera: An object-oriented software toolkit for chemical kinetics, thermodynamics, and transport processes. Caltech, Pasadena, $C A$,

Goodwin, G., Houim, R., \& Oran, E. (2016). Effect of decreasing blockage ratio on DDT in small channels with obstacles. Combustion and Flame, 173, 16-26. doi:https://doi.org/10.1016/j.combustflame.2016.07.029.

Khokhlov, A., Oran, E., \& Thomas, G. (1999). Numerical simulation of deflagration-to-detonation transition: the role of shock-flame interactions in turbulent flames. Combustion and Flame, 117, 323-339. doi:https ://doi .org/10.1016/S0010-2180 (98) $00076-5$.

Krivosheyev, P., Penyazkov, O., \& Sakalou, A. (2020). Analysis of the final stage of flame acceleration and the onset of detonation in a cylindrical tube using high-speed stereoscopic imaging. Combustion and Flame, 216, 146-160. doi:https://doi.org/10.1016/j.combustflame. 2020.02 .027$.

Masson-Delmotte, V., Zhai, P., Pörtner, H., Roberts, D., Skea, J., Shukla, P., Pirani, A., Moufouma-Okia, W., Péan, C., Pidcock, R. et al. (2018). IPCC, 2018: Summary for policymakers. Global Warming of $1.5^{\circ}$ C Above Pre-industrial Levels and Related Global Greenhouse Gas Emission Pathways, in the Context of Strengthening the Global Response to the Threat of Climate Change, Sustainable Development, and Efforts to Eradicate Poverty, . doi:http://pure.iiasa.ac .at/15716.

Murray, S. B. (2008). Numa manson on velocity deficits and detonation stability. Shock Waves, 18, 255. doi:https://doi.org/10.1007/ s00193-008-0128-z.

Oran, E., Gamezo, V., \& Khokhlov, A. (2002). Effects of boundary layers and wakes on shock-flame interactions and DDT. In 40th AIAA Aerospace Sciences Meeting \& Exhibit (p. 776). doi:https://doi.org/10.2514/6.2002-776.

Ren, K., Kotchourko, A., Lelyakin, A., \& Jordan, T. (2017). Numerical reproduction of DDT in small scale channels. In 201725 th International Conference on Nuclear Engineering. American Society of Mechanical Engineers Digital Collection. doi:https://doi.org/10.1115/ ICONE25-67150.

Teodorczyk, A., Drobniak, P., \& Dabkowski, A. (2009). Fast turbulent deflagration and DDT of hydrogen-air mixtures in small obstructed channel. international journal of hydrogen energy, 34, 5887-5893. doi:https://doi .org/10.1016/j . i jhydene.2008.11.120.

Thomas, G., Bambrey, R., \& Brown, C. (2001). Experimental observations of flame acceleration and transition to detonation following shock-flame interaction. Combustion Theory and Modelling, 5, 573-594. doi:https://doi.org/10.1088/1364-7830/5/4/304.

Tsugé, S. (1971). The effect of boundaries on the velocity deficit and the limit of gaseous detonations. Combustion Science and Technology, 3 , 195-205. doi:https://doi.org/10.1080/00102207108952287.

Yanez, J., \& Kuznetsov, M. (2016). Experimental study and theoretical analysis of a 'strange wave'. Combustion and Flame, 167, 494-496. doi:https://doi.org/10.1016/j.combustflame.2016.02.004.

Zel'dovich, Y. B., Gel'Fand, B., Kazhdan, Y. M., \& Frolov, S. (1987). Detonation propagation in a rough tube taking account of deceleration and heat transfer. Combustion, Explosion and Shock Waves, 23, 342-349. doi:https : //doi .org/10.1007/BF00748797.

Zhao, Y., Wang, C., \& Bi, Y. (2017). LES of flame acceleration and DDT in small-scale channels. Journal of Loss Prevention in the Process Industries, 49, 745-752. doi:https://doi.org/10.1016/j.jlp.2017.02.011. 